\title{
Sandor Type Inequalities for Sugeno Integral Based on s-Convex Function in the Second Sense
}

\author{
Lanping Li \\ School of Mathematics and Statistics, Hunan University of Finance and Economics, Changsha, China
}

Email address:

lilanping1981@163.com

To cite this article:

Lanping Li. Sandor Type Inequalities for Sugeno Integral Based on s-Convex Function in the Second Sense. Mathematics Letters. Vol. 4, No. 1, 2018, pp. 14-19. doi: 10.11648/j.ml.20180401.13

Received: March 1, 2018; Accepted: March 27, 2018; Published: May 4, 2018

\begin{abstract}
Integral inequalities play important roles in the fields of measure theory, probability theory and control theory. The aim of this paper is to develop some fuzzy integral inequalities. Sugeno integral is one of the most important fuzzy integrals, which has many applications in various fields. This paper constructs some new Sandor type inequalities for the Sugeno integral based on s-convex function in the second sense. Numerical examples are used to illustrate the effectiveness and practicality of the new inequalities.
\end{abstract}

Keywords: Fuzzy Integral, Sugeno Integral, Sandor Inequality, s-Convex Function

\section{Introduction}

There exists much vague or fuzzy information in the world. For example, one cannot or hardly give an accurate score for some delicious diet when facing much delicious food. The temperature in a room cannot be measured exactly because of the fluctuation [1]. In these situations, crisp numbers cannot work well. To overcome this shortcoming, professor Zadeh firstly introduce the concept of fuzzy set in 1965. Now, fuzzy set has accepted by people in various fields, also it has received great attention and applications in various fields [2-4]. At present, the theory and application of Zadeh's fuzzy set and some extensions, i.e. vague set, intuitionistic fuzzy set, hesitant fuzzy set, etc. have been utilized to solve a management decision and engineering problems [5-9].

Doctor Sugeno first proposed fuzzy integral in 1974, and since then Sugeno integral becomes an important analytical tool for measuring uncertain information [10-12]. Many scholars studied various fuzzy inequalities based on Sugeno integral and other fuzzy integrals. For example, Agahi et al. [13] established fuzzy Berwald type inequality for the Sugeno integral based on a concave function. Hosseini et al. [14] derived several versions of Hermite-Hadamard type inequality for pseudo-fractional integrals. Caballero and Sadarangani [15] derived several Hermite-Hadamard type inequalities for fuzzy integrals. For more details, one can see the references [16-21].

Sandor inequalities are important integral inequality for convex functions. Various results are derived for Sandor inequalities based on various type convex functions. Most of extended Sensor type inequalities are established based on ordinal definite integrals. In 1990, Sándor [22] first introduced the Sandor type inequality for definite integrals based on convex function. Caballero and Sadarangani [23] developed Sandor type inequalities for fuzzy integral with respect to ordinary convex function. For Sugeno integral, Li et al. [24] and Yang et al. [25] derived Sandor type inequalities with respect to general $(\alpha, \mathrm{m}, \mathrm{r})$-convex functions and $(\alpha, \mathrm{m})$ convex function, respectively.

S-convex function contains two types: the first and the second sense function of s-convex function. They are both important convex functions, which received great attention $[26,27]$. Under definite integral, many authors are interested in building inequality for this function. In this paper, we will extend the Sandor type inequality for s-convex function in the second sense for Sugeno integral. The construction of this article is as follows: Second 2 will recall the concepts and properties of Sugeno integral and s-convex function in the second sense. Section 3 will establish some new Sandor type inequalities for s-convex function in the second sense based on Sugeno integral. Finally, conclusions are provided in Section 4. 


\section{Preliminaries}

In this section, we will introduce some definitions and properties of Sugeno integral and s-convex function in the second sense. Here we always denote that $R$ is the real numbers set, $X$ is a nonempty set, $\Sigma$ is a $\sigma$-algebra of subsets of $X$ and $R_{+}=[0, \infty)$.

Definition 1 [28]. Let $\mu: \Sigma \rightarrow R_{+}$is a non-negative set function, we call it a non-additive measure, if it satisfies the following properties:

(i) $\mu(\Phi)=0$.

(ii) $A, B \in \Sigma$ and $A \subset B \Rightarrow \mu(A) \leq \mu(B)$.

(iii) For all $n \geq 1, A_{n} \in \Sigma$ and $A_{1} \subset A_{2} \subset \cdots$, $\Rightarrow$ $\mu\left(\bigcup_{n=1}^{\infty} A_{n}\right)=\lim _{n \rightarrow \infty} \mu\left(A_{n}\right)$.

(iv) For all $n \geq 1, A_{n} \in \Sigma, A_{1} \supset A_{2} \supset \cdots$ and $\mu\left(A_{1}\right)<+\infty$, $\Rightarrow \mu\left(\bigcap_{n=1}^{\infty} A_{n}\right)=\lim _{n \rightarrow \infty} \mu\left(A_{n}\right)$.

Remark 1. The triple $(X, \Sigma, \mu)$ is called a fuzzy measure space. For $\forall \alpha \geq 0$, we denote

$$
F_{\alpha}=\{x \mid x \in X, f(x) \geq \alpha\}=\{f \geq \alpha\} .
$$

Then if $\alpha \leq \beta$, we have $F_{\beta} \subset F_{\alpha}$.

Definition 2 [28]. Suppose that $(X, \Sigma, \mu)$ is a fuzzy measure space, $f: X \rightarrow R_{+}$be a non-negative measurable function, $A \in \Sigma$. The Sugeno integral of function $f$ defined on set $A$ is

$$
(S) \int_{A} f d \mu=\underset{\alpha \geq 0}{\vee}\left[\alpha \wedge \mu\left(A \cap F_{\alpha}\right)\right]
$$

Particularly, when $A=X$, the

$$
(S) \int_{X} f d \mu=\underset{\alpha \geq 0}{\vee}\left[\alpha \wedge \mu\left(F_{\alpha}\right)\right]
$$

Here, the operations $\vee$ and $\wedge$ are sup and inf on $R_{+}$, respectively.

Proposition 1 [28]. Suppose that $(X, \Sigma, \mu)$ be a fuzzy measure space, $A, B \in \Sigma$, and $f$ and $g$ are two non-negative measurable functions. Then

(i) $(S) \int_{A} f d \mu \leq \mu(A)$

(ii) For any positive real number $k$, we have

$$
(S) \int_{A} k d \mu \leq k \wedge \mu(A) \text {. }
$$

(iii) If the functions $f$ and $g$ satisfy:

$f \leq g$ on the set $A$,

Then

$$
(S) \int_{A} f d \mu \leq(S) \int_{A} g d \mu .
$$

(iv) If $A, B \in \Sigma, A \subset B$, then

$$
(S) \int_{A} f d \mu \leq(S) \int_{B} f d \mu .
$$

(v) If $\mu(A \cap\{f \geq \alpha\}) \geq \alpha$, then

$$
\text { (S) } \int_{A} f d \mu \geq \alpha .
$$

(vi) If $\mu(A \cap\{f \geq \alpha\}) \leq \alpha$, then $(S) \int_{A} f d \mu \leq \alpha$.

(vii) $(S) \int_{A} f d \mu<\alpha \Leftrightarrow$

There exists $\gamma<\alpha$, subject to $\mu(A \cap\{f \geq \gamma\})<\alpha$.

(ix) $(S) \int_{A} f d \mu>\alpha \Leftrightarrow$

There exists $\gamma>\alpha$, subject to $\mu(A \cap\{f \geq \gamma\})>\alpha$.

(x) If $\mu(A)<+\infty$, then

(S) $\int_{A} f d \mu \geq \alpha$ if and only if $\mu(A \cap\{f \geq \alpha\}) \geq \alpha$.

Remark 2. Let $F(\alpha)=\mu(A \bigcap\{f \geq \alpha\})$ then $F(\alpha)$ is call the distribution function of $f$ on $A$. According to the properties (v) and (vi) of Proposition 1, wecan get

$$
F(\alpha)=\alpha \Rightarrow(S) \int_{A} f d \mu=\alpha
$$

Then from a numerical point of view, the fuzzy integral (1) can be calculated as the solution of the equation $F(\alpha)=\alpha$.

Definition 3 [26]. Let $s \in(0,1]$ be a real number. A function $f: I \subseteq R_{+} \rightarrow R$ is said to be s-convex in the second sense if

$$
f(\lambda x+(1-\lambda) y) \leq \lambda^{s} f(x)+(1-\lambda)^{s} f(y)
$$

holds for all $(x, y) \in I$ and $\lambda \in[0,1]$.

Remark 3. Denote by $K_{s}^{2}$ the set of all s-convex functions in the second sense. If $\mathrm{s}=1$, then $K_{s}^{2}$ is ordinary convex function.

Lemma1 [29]. Let $x, y \geq 0$, then the inequality

$$
(x+y)^{\theta} \leq x^{\theta}+y^{\theta}
$$

holds for $\theta \in(0,1]$.

\section{Sandor Type Inequalities for Sugeno Integral Based on s-Convex in the Second Sense}

Sandor Type inequality provides estimates of the mean 
value of a nonnegative and convex function $f:[a, b] \rightarrow R$ with the following inequality $([30,31])$

$$
\frac{1}{b-a} \int_{a}^{b} f^{2}(x) d x \leq \frac{1}{3}\left[f^{2}(a)+f(a) f(b)+f^{2}(b)\right] .
$$

Unfortunately, Example 1 shows that Sandor type inequality with the form (7) is not valid for Sugeno integral based on s-convex functions in the second sense.

Example 1. Let $X=[0,1]$ and $\mu$ be the Lebesgue measure on $X$. If we take $f(x)=x$, then it is obvious that $f(x) \in K_{s}^{2}$. Calculate the Sugeno integral $(S) \int_{0}^{1} f^{2} d \mu$ by Remark 2, we get

$$
(S) \int_{0}^{1} f^{2} d \mu=\frac{3-\sqrt{5}}{2} \approx 0.382
$$

On the other hand,

$$
\frac{1}{3}\left[f^{2}(0)+f(0) f(1)+f^{2}(1)\right] \approx 0.3333
$$

This proves that the Sandor type inequality (6) is not satisfied for Sugeno integral with respect to s-convex function in the second sense.

In this section, we will derive some new Sandor type inequalities for the Sugeno integral with respect to s-convex function in the second sense.

Theorem 1. Let $f:[0,1] \rightarrow[0, \infty)$ is a s-convex function in the second sense, $s \in(0,1]$, and $\mu$ be the Lebesgue measure on $R$, then

$$
(S) \int_{a}^{b} f^{2} d \mu \leq \min \{\beta, 1\}
$$

where $\beta$ is the positive real solution of the equation

$$
1-\left(\frac{\sqrt{\beta}-f(0)}{f(1)}\right)^{1 / s}=\beta .
$$

Proof. Since $f(x) \in K_{s}^{2}$ for $x \in[0,1]$, then

$$
\begin{aligned}
f(x) & =f((1-x) \cdot 0+x \cdot 1) \\
& \leq(1-x)^{s} f(0)+x^{s} f(1)
\end{aligned}
$$

According to $(1-x)^{s} \leq 1, \forall x \geq 0$, we have

$$
f(x) \leq f(0)+x^{s} f(1)=g(x)
$$

According to (10) and the non-negative assumption of function $f(x)$, we have $f^{2}(x) \leq g^{2}(x)$ for all $x \in[a, b]$.

Then according to (iii) of Proposition 1 and Definition 2, we have

$$
\text { (S) } \int_{0}^{1} f^{2}(x) d \mu \leq(S) \int_{0}^{1} g^{2}(x) d \mu,
$$

$$
(S) \int_{0}^{1} g^{2}(x) d \mu=\underset{\beta \geq 0}{\vee}\left[\beta \wedge \mu\left([0,1] \cap\left\{g^{2} \geq \beta\right\}\right)\right]
$$

In order to calculate the Sugeno integral $(S) \int_{0}^{1} g^{2}(x) d \mu$, we need consider the distribution function $F$ of $g^{2}(x)$ on interval $[0,1]$ which is given as follows

$$
F(\beta)=\mu\left([0,1] \cap\left\{g^{2} \geq \beta\right\}\right)
$$

That is

$$
F(\beta)=\mu\left([0,1] \cap\left\{x \mid f(0)+x^{s} f(1) \geq \sqrt{\beta}\right\}\right)
$$

Then

$$
\begin{aligned}
F(\beta) & =\mu\left([0,1] \cap\left\{x \mid x \geq\left(\frac{\sqrt{\beta}-f(0)}{f(1)}\right)^{1 / s}\right\}\right) \\
& =1-\left(\frac{\sqrt{\beta}-f(0)}{f(1)}\right)^{1 / s}
\end{aligned}
$$

Let $F(\beta)=\beta$, then

$$
1-\left(\frac{\sqrt{\beta}-f(0)}{f(1)}\right)^{1 / s}=\beta .
$$

By (i) of Proposition 1 and (14), we have

$$
(S) \int_{0}^{1} f^{2}(x) d \mu \leq(S) \int_{0}^{1} g^{2}(x) d \mu=\min \{\beta, 1\}
$$

This completes the proof.

Now, we will prove the general case of Theorem.

Theorem 2. Let $f:[a, b] \rightarrow[0, \infty)$ is a s-convex function in the second sense, $s \in(0,1], f(b)>f(a)$ and $\mu$ be the Lebesgue measure on $R$, then

$$
\text { (S) } \int_{a}^{b} f^{2} d \mu \leq \min \{\beta, b-a\}
$$

where $\beta$ is the positive real solution of the equation

$$
(b-a)\left[1-\left(\frac{\sqrt{\beta}-f(a)}{f(b)}\right)^{1 / s}\right]=\beta
$$

Proof. Since $f(x) \in K_{s}^{2}$ for $x \in[a, b]$, then 


$$
\begin{aligned}
f(x) & =f\left(\left(1-\frac{x-a}{b-a}\right) a+\left(\frac{x-a}{b-a}\right) b\right) \\
& \leq\left(1-\frac{x-a}{b-a}\right)^{s} f(a)+\left(\frac{x-a}{b-a}\right)^{s} f(b)
\end{aligned}
$$

According to the fact that

$$
\left(1-\frac{x-a}{b-a}\right)^{s} \leq 1, \text { for any } x \in[a, b], s \in(0,1] \text {. }
$$

We have

$$
f(x) \leq f(a)+\left(\frac{x-a}{b-a}\right)^{s} f(b)=g(x),
$$

Combining (11) with the non-negative assumption of function $f(x)$, we have $f^{2}(x) \leq g^{2}(x)$ for all $x \in[a, b]$.

Then by (iii) of Proposition 1 and Definition 2, we have

$(S) \int_{a}^{b} f^{2}(x) d \mu \leq(S) \int_{a}^{b} g^{2}(x) d \mu$,

$$
(S) \int_{a}^{b} g^{2}(x) d \mu=\underset{\beta \geq 0}{\vee}\left[\beta \wedge \mu\left([a, b] \cap\left\{g^{2} \geq \beta\right\}\right)\right]
$$

To calculate the integral $(S) \int_{a}^{b} g^{2}(x) d \mu$, we consider the distribution function $F$ of $g^{2}(x)$ on $[a, b]$ which is given as follows

$$
F(\beta)=\mu\left([a, b] \cap\left\{g^{2} \geq \beta\right\}\right)
$$

That is

$$
\begin{aligned}
F(\beta) & =\mu\left([a, b] \cap\left\{x \mid f(a)+\left(\frac{x-a}{b-a}\right)^{s} f(b) \geq \sqrt{\beta}\right\}\right) \\
& =\mu\left([a, b] \cap\left\{x \mid x \geq a+(b-a)\left(\frac{\sqrt{\beta}-f(a)}{f(b)}\right)^{1 / s}\right\}\right) \\
& =(b-a)\left[1-\left(\frac{\sqrt{\beta}-f(a)}{f(b)}\right)^{1 / s}\right]
\end{aligned}
$$

Let $F(\beta)=\beta$, then

$$
(b-a)\left[1-\left(\frac{\sqrt{\beta}-f(a)}{f(b)}\right)^{1 / s}\right]=\beta .
$$

By (i) of Proposition 1 and (21), we obtained

$$
(S) \int_{a}^{b} f^{2}(x) d \mu \leq(S) \int_{a}^{b} g^{2}(x) d \mu=\min \{\beta, b-a\}
$$

Then we complete the proof.

To show the valid and effectiveness of the above established Sandor type fuzzy inequalities, we see the following example.

Example 2. Let $X=[a, b]$ and $\mu$ be the Lebesgue measure on $X$. If we take the function $f(x)=x$, then by Remark 3, we know that $f(x) \in K_{s}^{2}$ for $s=1$.

(i) For the case $[a, b]=[0,1]$, by solving the equation

$$
(1-0)\left[1-\left(\frac{\sqrt{\beta}-f(0)}{f(1)}\right)\right]=\beta
$$

We get

$$
\beta=\frac{3-\sqrt{5}}{2} \approx 0.3820
$$

Straightforward calculation shows that

$$
\text { (S) } \int_{0}^{1} f^{2} d \mu=\frac{3-\sqrt{5}}{2} \approx 0.3820 \text {. }
$$

Comparing the equations (23) with (24), we can see the following result

$$
(S) \int_{0}^{1} f(x) d \mu=\min \{\beta, 1-0\} .
$$

(i) For the case $[a, b]=[2,3]$, by solving the equation

$$
(3-2)\left[1-\left(\frac{\sqrt{\beta}-f(2)}{f(3)}\right)\right]=\beta
$$

We get

$$
\beta=1.2883
$$

Straightforward calculation shows that

$$
(S) \int_{2}^{3} f^{2} d \mu \approx 0.5892
$$

Comparing the equations (27) with (28), we can see the following result

$$
(S) \int_{2}^{3} f(x) d \mu \leq \min \{\beta, 3-2\} .
$$

This also implies the fuzzy Sandor inequalities can get a well estimate of Sugeno integral $(S) \int_{0}^{1} f^{2} d \mu$.

\section{Conclusions}

The study of integral inequality is an important topic, which has been attention by many scholars. Recently, fuzzy set and fuzzy integral achieve great success in many fields. Sandor type integral inequality provides estimates of the mean value of a nonnegative and ordinary convex function. This paper intends to establish an upper approximation for 
the Sugeno integral of s-convex functions in the second sense based on ordinary Sandor type inequalities. Several examples are provided to illustrate the validity of this inequality. In the future study, we will study the other properties of s-convex functions in the second sense for some other fuzzy integrals.

\section{Acknowledgements}

This study is partially supported by 933rd items of Education Reform Project in Hunan Province in 2016. The author is grateful to the reviewers for a very careful reading of the manuscript and the suggestions which lead to the improvement of the paper.

\section{References}

[1] Wu H C. Fuzzy Bayesian estimation on lifetime data [J]. Computational Statistics, 2004, 19 (4):613.

[2] Dubois D, Prade H, Esteva F, et al. Fuzzy set modelling in case-based reasoning [J]. International Journal of Intelligent Systems, 2015, 13 (4):345-373.

[3] Fan B, Tsang E C C, Xu W, et al. Double-quantitative rough fuzzy set based decisions [J]. Information Sciences, 2017, 378 (C):264-281.

[4] Ezghari S, Zahi A, Zenkouar K. A new nearest neighbor classification method based on fuzzy set theory and aggregation operators [J]. Expert Systems with Applications, 2017, 80:58-74

[5] Chen S M, Cheng S H, Chiou C H. Fuzzy multiattribute group decision making based on intuitionistic fuzzy sets and evidential reasoning methodology $[\mathrm{J}]$. Information Fusion, 2016, 27:215-227.

[6] Ye Y, Liang L, Cao Y, et al. Optimization and sorting method of the engineering layout scheme for interconnected river system network based on vague set and cloud model [J]. System Engineering Theory \& Practice, 2017, 37 (7):1926-1936.

[7] Zhu B, Xu Z, Xu J. Deriving a ranking from hesitant fuzzy preference relations under group decision making [J]. IEEE Transactions on Cybernetics, 2017, 44 (8):1328-1337.

[8] Chen T Y. The inclusion-based TOPSIS method with intervalvalued intuitionistic fuzzy sets for multiple criteria group decision making [J]. Applied Soft Computing, 2015, 26:57-73.

[9] Dong J, Wan S. A new method for multi-attribute group decision making with triangular intuitionistic fuzzy numbers [J]. Kybernetes, 2016, 45 (1):158-180.

[10] Saito K, Notomi K, Hashimoto H and Saito M. Application of the Sugeno integral with $\lambda$-fuzzy measures to endoscopic diagnosis. Biomedical Fuzzy \& Human Sciences the Official Journal of the Biomedical Fuzzy Systems Association, 2017, 9 (1): 11-16.

[11] Melin P, Mendoza O, Castillo O. Face recognition with an improved interval type-2 fuzzy logic Sugeno integral and modular neural networks [J]. IEEE Transactions on Systems, Man, and Cybernetics - Part A: Systems and Humans, 2011, 41 (5):1001-1012.
[12] Daraby B, Asll H G, Sadeqi I. General related inequalities to Carlson-type inequality for the Sugeno integral [J]. Applied Mathematics \& Computation, 2017, 305:323-329.

[13] Agahi H, Mesiar R, Ouyang Y, et al. Berwald type inequality for Sugeno integral [J]. Applied Mathematics \& Computation, 2010, 217 (8):4100-4108.

[14] Hosseini M, Babakhani A, Agahi H, et al. On pseudofractional integral inequalities related to Hermite-Hadamard type [J]. Soft Computing, 2016, 20 (7):2521-2529.

[15] Caballero J, Sadarangani K. Hermite-Hadamard inequality for fuzzy integrals [J]. Applied Mathematics \& Computation, 2009, 215 (6):2134-2138.

[16] Abbaszadeh S, Gordji M E, Pap E, et al. Jensen-type inequalities for Sugeno integral [J]. Information Sciences, 2016, 376:148-157.

[17] Abbaszadeh S, Eshaghi M. A Hadamard-type inequality for fuzzy integrals based on $r$-convex functions [J]. Soft Computing, 2016, 20 (8):3117-3124.

[18] Agahi H, Babakhani A, Mesiar R. Pseudo-fractional integral inequality of Chebyshev type [J]. Information Sciences, 2015, 301 (C):161-168.

[19] Alzer H, Man K K. A Hardy-Littlewood integral inequality on finite intervals with a concave weight [J]. Periodica Mathematica Hungarica, 2015, 71 (2):184-192.

[20] Abbaszadeh S, Eshaghi M. A Hadamard-type inequality for fuzzy integrals based on r-convex functions $[\mathrm{J}]$. Soft Computing, 2016, 20 (8), 3117-3124.

[21] Latif M A, Irshad W, Mushtaq M. Hermite-Hadamard type inequalities for $\mathrm{m}$-convex and $(\mathrm{a}, \mathrm{m})$-convex functions for fuzzy integrals [J]. Journal of Computational Analysis \& Applications, 2018, 24 (3):497-506.

[22] Sándor J. On the identric and logarithmic means. Aequationes Mathematicae, 1990, 40 (1):261-270.

[23] Caballero J, Sadarangani K. Sandor's inequality for Sugeno integrals [J]. Applied Mathematics \& Computation, 2011, 218 (5):1617-1622.

[24] Li D Q, Cheng Y H, Wang X S. Sandor type inequalities for Sugeno integral with respect to general $(\alpha, \mathrm{m}, \mathrm{r})$-convex functions [J]. Journal of Function Spaces, 2015, 2015 (37):113.

[25] Yang X L, Song X Q, Lu W. Sandor's type inequality for fuzzy integrals [J]. Journal of Nanjing University, 2015, 32 (2):144-156

[26] Hudzik H, Maligranda L. Some remarks on s-convex functions [J]. Aequationes Mathematicae, 1994, 48 (1):100111.

[27] Kunt M. On New İnequalities of Hermite-Hadamard-Fejér Type for Harmonically s-Convex Functions via Fractional İntegrals [J]. Applied Mathematics \& Computation, 2016, 259 (1):875-881.

[28] Z. Wang, G. Klir, Fuzzy Measure Theory [M], Plenum, New York, 1992.

[29] Ren H, Wang G, Luo L. Sandor type fuzzy inequality based on the $(\mathrm{s}, \mathrm{m})$-convex function in the second sense [J]. Symmetry, 2017, 9 (9):181-190. 
[30] Lu W, Song X Q, Huang L L. Inequalities of HermiteHadamard and Sandaor for fuzzy integral [J]. Journal of Shandong University (Natural Science), 2016, 51 (8): 22-28.
[31] Yang Y Y, Qian W M. Two optimal inequalities related to the Sandor-Yang type mean and one-parameter mean [J]. Communications in Mathematical Research, 2016, 32 (4):352358 . 